\title{
M. Lindsay KAPLAN, Figuring Racism in Medieval Christianity
}

\section{Pierre Levron}

\section{(2) OpenEdition}

\section{Journals}

Édition électronique

URL : https://journals.openedition.org/ccm/5390

DOI : $10.4000 / \mathrm{ccm} .5390$

ISSN : 2119-1026

\section{Éditeur}

Centre d'études supérieures de civilisation médiévale/Université de Poitiers

\section{Édition imprimée}

Date de publication : 1 décembre 2020

Pagination : 283-286

ISBN : 978-2-490783-07-6

ISSN : 0007-9731

\section{Référence électronique}

Pierre Levron, «M. Lindsay KAPLAN, Figuring Racism in Medieval Christianity», Cahiers de civilisation médiévale [En ligne], 252 | 2020, mis en ligne le 02 décembre 2020, consulté le 02 décembre 2022. URL : http://journals.openedition.org/ccm/5390 ; DOI : https://doi.org/10.4000/ccm.5390

\section{(c) $(1) \odot$}

Creative Commons - Attribution - Pas d'Utilisation Commerciale - Pas de Modification 4.0 International - CC BY-NC-ND 4.0

https://creativecommons.org/licenses/by-nc-nd/4.0/ 
M. Lindsay Kaplan, Figuring Racism in Medieval Christianity, New York, Oxford University Press, 2019.

M. Lindsay Kaplan assigne à son Figuring Racism in Medieval Christianity (désormais FRMC) deux buts : étudier la genèse de représentations racistes visant en premier lieu les juifs et ensuite les noirs, et décrire le système de «pensée par figures» centré sur les figures de Chanaan, de Ham et d'Ismaël qui fonde l'idée d'une servitude perpétuelle des Juifs, servant de prototype puis de justification à la traite des noirs, initiée en Europe par les Portugais à partir des années $40 \mathrm{du} \mathrm{Xv} v^{\mathrm{e}} \mathrm{s}$. L'ouvrage se situe dans un courant historiographique - mais aussi politique, l'a. justifiant son travail par la nécessité de combattre le racisme et les groupes suprémacistes blancs des ÉtatsUnis dans sa coda centrée sur la thèse des origines communes de l'antisémitisme et du racisme représentés en Europe par Dik Van Arkel (The Drawing of the Marks of Caïn: A Socio-Historical Analysis of the Growth of Anti-Jewish Stereotypes, Amsterdam, Amsterdam University Press, 2009), le mouvement du Moyen Âge à la modernité caractéristique de FRMC se rencontrant aussi bien chez D. Van Arkel que chez Lynn Tarte Ramey (Black Legacies: Race and the European Middle Ages, Gainesville, University Press of Florida, 2014). Le présent compte rendu traitera trois axes : il commencera par une présentation rapide des chapitres de l'ouvrage, avant d'en examiner les résultats et de discuter des points qui susciteront les critiques et les débats.

FRMC possède donc cinq chapitres. Le premier examine le concept de la servitus judaeorum, appuyé sur Matthieu, 27:25 et passant de la théologie au droit canon; le deuxième envisage une caractérisation médicale des Juifs sous l'influence de la bile noire, affectés de saignements anaux analogues aux menstruations; 
le troisième aborde le volet iconographique du travail de l'a. : la présence du motif de persécuteurs juifs à peau sombre dans deux psautiers anglais du début du XIII ${ }^{\mathrm{e}}$ s. : les Psautiers des ms. Londres, British Library Royal I D X, et Londres, British Library, Arundel 157; le quatrième chapitre étudie les commentaires de la malédiction que Noé, dans la Genèse, adresse à Ham, et qui fonde la représentation «par figures» de la servitude des Juifs; le cinquième traite des fondements juridiques (dans le droit canon, via les bulles papales) de l'asservissement et de la traite des Africains. Ces deux derniers chapitres ont des développements consacrés au début de la période moderne, abordée sous l'angle de la continuité avec l'époque médiévale.

Que résulte-t-il de l'examen de cet ouvrage? FRMC se situe, tout d'abord, dans un courant historiographique et intellectuel qui veut combattre les représentations racistes existant dans les sociétés contemporaines en en faisant l'étiologie. Il s'oppose en cela à la notion d' «altérité» non ethnicisée qui caractérise les études médiévales françaises, et dont Francis Dubost (Aspects fantastiques de la littérature narrative médiévale [XII ${ }^{e}$ XIII ${ }^{e}$ s.] : l'autre, l'ailleurs, l'autrefois, Paris, Honoré Champion [Nouvelle bibliothèque du Moyen Âge, 15], 1991) ou Jean-Claude Vallecalle (Messages et ambassades dans l'épopée française médiévale : l'illusion du dialogue, Paris, Honoré Champion [Nouvelle bibliothèque du Moyen Âge, 82], 2006) sont deux importants représentants. Si une tension préjudiciable à la qualité de l'apport scientifique de l'ouvrage se fait jour entre ses visées historiographiques et ses buts politiques, le principal mérite de FRMC est de soulever un débat entre les écoles caractéristiques de la médiévistique française et les tendances d'origine anglo-saxonne. Il met en évidence aussi l'existence de deux conceptions des origines des «races » dans ces courants anglo-saxons. Si l'ouvrage de L. Tarte Ramey en situe l'origine dans des modèles scientifiques, M. Lindsay Kaplan la situe dans la théologie chrétienne médiévale, et plus précisément dans son aspect de polémique contra judaeorum. Il présente par ailleurs quelques résultats importants. Il y a tout d'abord l'idée d'un racisme anti-Noirs dérivant de l'antisémitisme. Il y a aussi l'idée d'une «pensée par figures» qui, en se transférant de la théologie au droit mais concernant aussi l'histoire de l'art ou la médecine, contribue à l'approche de la pensée médiévale, et complète en partie l'approche de Florence Goyet (Penser sans concepts : fonctions de l'épopée guerrière. Iliade, Chanson de Roland, Hôgen et Heiji Monogatari, Paris, Honoré Champion
[Bibliothèque de littérature générale et comparée, 61], 2006) décrivant l'épopée comme un moyen de penser sans concepts, même s'il serait vraiment nécessaire d'approfondir l'étude de ce système, le recours à Erich Auerbach demeurant indispensable. L'ouvrage permet cependant d'observer le mouvement suivant : 1) Définition, à partir de la Genèse et des Psaumes, de figures vétérotestamentaires de l'infériorité religieuse; elles peuvent être représentées dans l'iconographie; 2) ces figures servent à la définition de modèle juridique en droit canon; 3 ) Ce système théologique et juridique influence la réflexion sur la guerre juste et sur le droit à asservir les captifs à la fin du Moyen Âge; 4) appliqué tout d'abord aux juifs, ce système concerne ensuite les musulmans et les Africains. FRMC a, de ce point de vue, le mérite de présenter un grand nombre de textes-relais relevant de la théologie et du droit. La période comprise entre la fin $d u \mathrm{XII}^{\mathrm{e}} \mathrm{s}$. et les trois premières décennies du siècle suivant sont celles où les parties théologiques, juridiques et iconographiques de la question se mettent en place. L'usage que l'a. fait de la bulle papale Etsi Judaeos de 1205 est aussi à mettre au compte de FRMC, l'ouvrage de D. Van Arkel n'abordant jamais ce texte. Les contenus juridiques permettent de décrire un élément de la différenciation progressive du droit canon par rapport au droit romain : la figure devient la base d'un droit à valeur théologique, tandis qu'il est possible de reconstituer une évolution qui part de la prohibition des conversions forcées par les textes grégoriens de 591 et de 602 pour aboutir, à partir du commentaire de la De summa casibus de Guillaume de Rennes à un corpus juridique partant de la décrétale Qui Sincera et incluant Dispar nimirum et Etsi Judaeos. Un autre ensemble de données s'est révélé être peut-être la plus grande innovation de $F R M C$ : les contenus médicaux de cette infériorisation des juifs. L'assimilation des Juifs à un tempérament atrabilaire apparaît donc dans la réflexion médicale de la fin du XIII ${ }^{\mathrm{e}} \mathrm{s}$. Les apports relèvent de l'épistémologie, dans la mesure où ce chapitre 2 permet de décrire un transfert culturel partant de la théologie et du droit canon pour atteindre la médecine, celle-ci se définissant comme une science exploitant des connaissances classiques, mais voulant devenir un modèle de science chrétienne. Le motif du «saignement juif» participe à la transformation d'une médecine «post-classique» en médecine pleinement médiévale; il sert de synthèse à la théologie et à la médecine. Il démontre également la puissance d'assimilation de la notion de mélancolie. L'histoire culturelle est le second apport de ce chapitre 2, qui met en évidence un rôle polémique de la mélancolie que Jacqueline Cerquiglini-Toulet (La couleur de la 
mélancolie : la fréquentation des livres au XIV siècle, 1300-1400, Paris, Hatier [Brèves littératures], 1991) n'a pas abordé.

Quels sont les points plus discutables de FRMC? La lecture de l'ouvrage permet d'en mettre un certain nombre en évidence. Le plus important semble bien être la définition ethnicisée des peuples visés. L'approche a le défaut majeur d'entériner, sur le plan anthropologique mais aussi idéologique, des conceptions qui n'ont nullement lieu d'être d'un point de vue biologique. Il importe donc d'utiliser cet ouvrage avec de grandes précautions afin de ne pas se placer sous un angle trop proche de celui des adversaires que l'a. veut combattre. L'enjeu final d'un discours politique brouille la destination réelle du livre, qui risque de se réduire à l'histoire de la construction d'un discours d'autorité réputé encore valoir de nos jours et contre lequel il faut bâtir des contre-discours concernant nos sociétés actuelles. Tout aussi gênante pour un usage scientifique de cet ouvrage est une construction qui paraît rigoureuse et qui produit une synthèse assez efficace des questions traitées mais qui présente de nombreux défauts. L'écriture d'histoire - dont les ex. les plus anciens sont les Antiquités juives de Flavius Josèphe et la General Estoria - aurait pu, chronologiquement parlant, s'insérer dans les mêmes temporalités que les sources théologiques et juridiques; elle n'intervient que dans le chapitre 5, dont les faiblesses générales de construction sont par ailleurs nombreuses. Il repose beaucoup sur une littérature secondaire dont certains auteurs - James Muldoon et A. C. de C. M. Saunders en particulier - sont très largement exploités, donnant l'impression que cette partie de l'ouvrage est une synthèse de leurs travaux davantage qu'une réflexion originale. Le texte a, d'une façon générale, une construction répétitive qui se met au service d'un discours trop facilement unificateur. C'est ainsi que le corpus romano-civiliste convoqué dans les chapitres 1 et 5 se limite à Florentinus, à Justinien et à Azon. L'argumentation oppose trois compilateurs ou professeurs de droit romain et civil à des auteurs canonistes, décrétistes ou décrétalistes bien plus nombreux. Si ce parti pris a une certaine logique, dans la mesure où il décrit une question dont les sources relèvent de la philosophie politique aristotélicienne et de l'augustinisme théologique, il tend à englober quelque peu abusivement l'ensemble des juristes médiévaux. Il aurait été préférable de confronter ces sources à celles provenant du droit civil et de ses enseignants ou commentateurs, et Franciscus Accursius en particulier, qui n'accorde aucune place à cette question. FRMC ne permet donc pas de se faire une idée des différences, voire des débats implicites, séparant deux sources médiévales essentielles de droit savant. Le développement du chapitre 5 consacré à la consultation du pape Eugène IV par le roi Duarte appuie son développement sur le seul avis d'Antonio Roselli. Rien n'est dit du second canoniste consulté, qui n'est même pas mentionné, alors qu'il aurait été utile de mentionner sa position. Le lecteur en est réduit à penser que sa réponse a été comparable, sans pouvoir le confirmer ou l'infirmer. L'argumentation du chapitre 3 souffre d'un défaut assez comparable. Elle ne tient pas compte tout d'abord de l'ambivalence des carnations sombres ou noires dans la culture médiévale. Aucune mention n'est ainsi faite de l'épouse nigra sed formosa du Cantique des Cantiques, pourtant très commentée, ni de la Reine de Saba, figure très positive. Les Vierges noires, dont la production iconographique se fait en Occident entre le $\mathrm{XI}^{\mathrm{e}}$ et le $\mathrm{XV}^{\mathrm{e}} \mathrm{s}$. et entre le XIII et le XIV ${ }^{e}$ s. dans le monde byzantin, auraient pu constituer un contre-pied efficace à la thèse défendue; elles n'apparaissent pas! Les Psautiers des manuscrits Paris, Bibliothèque de l'Arsenal 604, et Bibliothèque Sainte-Geneviève, 1273, datant tous les deux du XIII ${ }^{\mathrm{e}}$ s., montrent respectivement un roi noir à la fonction positive (Arsenal 604, fol. 256 verso) et un apôtre noir (BSG 1273, fol. 18 verso). Il est donc impossible de ranger cette couleur de peau dans la catégorie du négatif ou du néfaste, de nombreuses représentations iconographiques distinguant clairement les démons (qui peuvent avoir des peaux grises, bleues, marron ou noires) des êtres humains, dont le teint blanc/beige sera partagé par tous. Le dossier iconographique - bien mince! - paraît plutôt mettre en évidence des cas très particuliers, soumis à la concurrence de codes concurrents qui l'emportent massivement tels que les traits rendus difformes ou le port du chapeau à pointe. Les illustrations du ms. Londres, BL Sloane 346 auraient pu servir de support à un débat sur la pertinence de cet aspect dans le code iconographique. FRMC tend dès lors, d'une manière générale, à simplifier d'une manière qui se révèle particulièrement abusive des phénomènes complexes, et dont une étude scientifique pertinente exigerait une quantité bien plus étendue de sources primaires. L'a. ne tient en outre pas compte des dynamiques de transformation lourde des modèles intellectuels et des savoirs au cours du Moyen Âge, particulièrement sensible en ce qui concerne les connaissances «pré-ethnologiques » ou «pré-ethnographiques». La lecture révèle aussi d'autres éléments appelant de toute urgence une discussion critique : le terme de «païens » (pagans) employé par l'a. pour désigner les habitants des Canaries fait partie du lexique polémique que les théologiens chrétiens médiévaux, mais 
aussi les écrivains en langue vulgaire, utilisent quand ils combattent l'Islam, parlent de la religion gréco-romaine ou qualifient les Sarrasins des chansons de geste. Son emploi - qui simplifie le pro pos - est ennuyeux dès lors que l'on veut faire l'étiologie de conceptions racistes pour combattre le racisme dans la société actuelle. Une autre question, suscitée en particulier par les chap. 4 et 5, se pose. Qu'entendre par «populaire» quand l'on s'interroge sur un phénomène de la culture médiévale? Aucun élément de réponse n'est fourni. Les sources primaires font toutes partie d'une culture savante, et aucun élément ne permet de supposer l'existence de transferts dans la littérature de l'époque.

Il faut relever pour finir quelques lacunes bibliographiques sérieuses. La plus importante, eu égard à l'axe historiographique poursuivi par ces deux ouvrages, est l'absence de l'ouvrage de D. Van Arkel précité ; citons aussi, dirigé par KarlAugust Wirth, Pictor in Carmine: Ein Handbuch der Typologie aus der Zeit um 1200 nach ms. 300 des Corpus Christi College in Cambridge (Berlin, Gebrüder Mann [Veröffentichungen des
Zentralinstituts für Kunstgechichte, 17], 2006), de deux études de Gilbert Dahan (Les intellectuels chrétiens et les juifs au Moyen Âge, Paris, Cerf, [Patrimoines. Judaïsme], 1990; Le Brûlement du Talmud à Paris, 1242-1244 [en tant que directeur de la publication], Paris, Cerf [Nouvelle Gallia judaïca, 1], 1999) et de l'ouvrage d'Aron Iakovlevitch Gurevitch, Medieval Popular Culture: Problems of Belief and Perception (Cambridge, Cambridge in University Press [Cambridge Studies in Oral and Literate, Culture, 14], 1988).

FRMC est-il utilisable? La prépondérance quantitative des points faibles identifiés sur les résultats obtenus incite à une très grande prudence. Il est néanmoins informatif d'une tendance historiographique et militante d'école anglo-saxonne, et est donc doté d'un certain intérêt épistémologique. Mais il ne peut être considéré en aucun cas comme une étude de fond.

Pierre LEVRON

UMR 7302 - CESCM

Université de Poitiers 